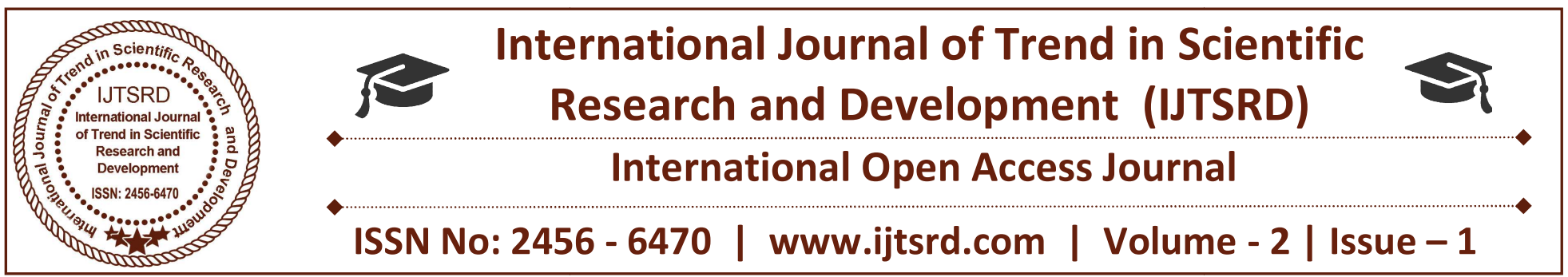

\title{
RAPD based Molecular Characterization of Lavatera Cachemiriana Cambess
}

\begin{abstract}
Mudasir A Mir
Centre for Plant Biotechnology, Division of

Biotechnology, Sher-e-Kashmir University of

Agricultural Sciences and Technology of Kashmir,

Shalimar, 191121, India
\end{abstract}

\author{
Amrina Shafi \\ Division of Biotechnology, CSIR-Institute of \\ Himalayan Bioresource Technology, Palampur, \\ Himachal Pradesh, 170761, India
}

\begin{abstract}
Insha Zahoor
Department of Biotechnology, School of Biological Sciences, University of Kashmir, Srinagar, Jammu and Kashmir, 190006, India
\end{abstract}

\section{ABSTRACT}

The exploration of genetic diversity is important to properly maintain and exploit germplasm resources and to design better strategically defined approaches in the utilization of collected germplsam so as to safeguard it from extinction. Therefore, current study was undertaken to evaluate genetic diversity of Lavatera cachemiriana collected from different geographical locations. RAPD based molecular markers were used to evaluate genetic diversity. Total DNA was isolated using CTAB method, molecular marker based polymorphism was carried out using ISSR and RAPD molecular markers, while as genetic analysis was determined in the form of RP, MI, PIC, EMR, Nei-Li's similarity matrix using different tools and techniques. A total number of 137 DNA fragments were obtained (average- 9.13/primer) which resulted from 15 RAPD primers, among these 33 were found to be monomorphic and rest as polymorphic. The highest polymorphic bands were generated by OPT06 (9) with percentage of polymorphism as $90 \%$. Three RAPD primers have showed number of polymorphic bands between 4-6 i.e. OPB10 (4), OPF03/OPG09 (6) with polymorphic percentage between $44.44 \%-75.0 \%$. PIC value ranged between $0-0.29$ with highest value shared by OPT06 (0.23). EMR was found between 0-6.80 with highest value shared by OPG09. Nei's genetic distance (NeiGD) and Shannon's Index (I) were found as 0.119 and 0.075 respectively. EMR was found between 0-6.80 with highest value shared by OPG09. Nei's genetic distance (NeiGD) and Shannon's Index (I) were found as 0.119 and 0.075 respectively. Two distinct clusters $(1 \& 2)$ were obtained which formed a major cluster at 0.98 level of similarity. The cluster 1 belongs to TANG and PAMP samples and showed closer genetic relationship with 0.996 level of similarity. Therefore, the results showed that Lavatera cachemiriana possesses lower but significant levels of polymorphism among collected samples.

Keywords: Lavatera kashmiriana, Genetic analysis, Molecular Characterization, Polymorphism

\section{INTRODUCTION}

The Himalayan region of India is an important repository which harbours rich germplasm with around 3,500 medicinal plant species. It is estimated that 17 million hectares of Indian forest land produces more than $80 \%$ of medicinal and aromatic plants. Due to rich floral wealth, India is emerging an integral part of global herbal market and could become largest raw material supplier of herbal drugs (Parveen, 2013). The state of Jammu and Kashmir of western Himalaya 
possesses about 2104 vascular plants that have acted as source of natural remedies. It is imperative for the current generation to scientifically explore floral diversity, design constructive strategies for sustainable utilization and conservation of forest flora, so as to conserve the natural heritage of flora for the future generations (Dar et al; 2002).

The evaluation of genetic diversity levels or molecular characterization is an important area for conservation management, sustainable utilization, genetic resource characterization and amelioration of any species, the genetic diversity in a species depends on various multitude of factors including geographic distribution, breeding system, gene flow, human intervention etc. (Loveless, 1984; Sundaram and Purwar 2011). Molecular markers are useful tool for taxonomic identification, genetic relationship and germplasm management of species. The various molecular markers can be used to assess molecular diversity based on their technical availability and can complement one another (Domyati et al., 2011) and PCR based methods are in demand due to simplicity and they need only small quantities of sample DNA (Bornet and Branchard, 2001). Random amplified polymorphic DNA (RAPD) is a PCR oriented technique and is fast, simple, using single oligonucleotide primer (8-10 nucleotides) with arbitrary sequence (Chikkaswamy and Prasad, 2012). Despite existing limitations of RAPD (Dominant inheritance mood), these markers have been used extensively to study genetic diversity $\&$ have been applied in gene mapping studies to fill gaps not covered by other markers. This is due to their various attributes such as simplicity, high speed, low cost, low DNA demand \& also no prior target DNA sequence information required (Marin et al., 2007; Domyati et al., 2011).

Lavatera cachemiriana Cambess (Malvaceae) an important medicinal herb native to Kashmir Himalayas. Traditionally it is used for range of medicinal purposes with therapeutic potential like laxative (Sharma, 2003), abdominal disorders, renal colic (Kaul, 2010), common cold and mumps, antiseptic etc. (Dar et al., 2002). It is known as sazakul in Kashmiri language or reshkhatmi in persian (Vidyarthi, 2010), while as different names are being used locally for various parts of this plant species such as sazposh for flowers, sazamool for the roots (Kaul, 1997; Vidyarthi, 2010) and wan sotsal for leaves (Dar et al, 2002). As a conservation management strategy, it is vital to assess genetic diversity within and among plant species because level of genetic variation assists them to adapt towards changing environmental conditions. Therefore, the current research work was carried out to evaluate existence of genetic diversity in sample accessions of Lavatera cachemiriana utilizing RAPD based molecular markers.

\section{MATERIALS AND METHODS}

\section{Sample Collection}

Leaf samples of Lavatera cachemiriana Cambess were collected from 5 geographically distinct locations of Kashmir Himalaya, India. The leaves were immediately immersed in liquid nitrogen and maintained at sub-zero temperature until DNA extraction. The samples were authenticated at Centre for Biodiversity and Taxonomy, University of Kashmir herbarium (KASH) and voucher specimen numbers were assigned accordingly.

\section{DNA extraction}

DNA was extracted using slightly modified CTAB method (Doyle and Doyle, 1987; Chikkaswamyand Prasad, 2012). Briefly, 1g leaf from each collected sample was grinded with liquid nitrogen separately and were transferred to tubes containing $6 \mathrm{ml}$ of extraction buffer (3\% CTAB, $100 \mathrm{mM}$ Tris, $20 \mathrm{mM}$ EDTA, $1.4 \mathrm{M} \mathrm{NaCl}, 2 \% \quad \mathrm{PVP}$ and $1 \% \quad \beta$ mercaptoethanol). The samples were incubated at $65^{\circ} \mathrm{C}$ for 1 hour with continuous shaking. The centrifuge tubes were spun at 7,000 rpm for $15 \mathrm{~min}$ at $4^{\circ} \mathrm{C}$. The supernatants were collected in fresh tubes, followed by addition of $6 \mathrm{ml}$ of chloroform and isoamyl alcohol (24:1). The contents were mixed well gently by inverting tubes for 25-30 times and again centrifuged at $7,000 \mathrm{rpm}$ for $15 \mathrm{~min}$ at $4{ }^{\circ} \mathrm{C}$. The supernatants were transferred to a fresh tube. The supernatants were kept overnight at $4^{\circ} \mathrm{C}$ to precipitate DNA by adding half a volume of $5 \mathrm{M} \mathrm{NaCl}$ and equal volume of iso-propanol followed by centrifugation at $12,000 \mathrm{rpm}$ for $20 \mathrm{~min}$. The pellets obtained were washed with $70 \%$ ethanol. The dried DNA pellets were dissolved in $0.5 \mathrm{ml}$ TE buffer $(10 \mathrm{mM}$ Tris- $\mathrm{HCl}$ $\mathrm{pH} 7.4,1 \mathrm{mM}$ EDTA), followed by addition of $5 \mu \mathrm{l}$ RNase A $(2 \mathrm{mg} / \mathrm{ml})$ and incubated for $60 \mathrm{~min}$ at $37^{\circ} \mathrm{C}$.

\section{DNA Quantification and purity check}

DNA was quantified using Uv-Vis spectrophotometer (Chemito Technologies (Ahmed et al., 2013). The 
concentration was measured at absorbance of $260 \mathrm{~nm}$ using below formula:

DNA concentration $(\mu \mathrm{g} / \mathrm{ml})=\mathrm{A}_{260} \mathrm{X} 50 \mathrm{X}$ Dilution factor

The purity and quality of extracted DNA was determined by measuring absorbance ratios as $260 \mathrm{~nm} / 280 \mathrm{~nm}$ along with electrophoresis on $0.8 \%$ $(\mathrm{w} / \mathrm{v})$ agarose gel using 1 x TBA buffer (Trisbase,boric acid, $0.5 \mathrm{M}$ EDTA) for $\sim 45$ minutes. The developed gel was visualized using Gel Documentation system (Vilber Lourmat, Germany) and obtained DNA was stored at $-20^{\circ} \mathrm{C}$ for further downstreaming.

\section{Polymerase chain reaction amplification (PCR amplification)}

RAPD primers (20 arbitrary/10-mer) of group A, B, C, D, E, F, G, M, N, T (Operon Technology, USA) were initially screened for detection of DNA polymorphism. Each PCR amplification was performed in a final volume of $15 \mu$ reaction mixture containing 10-25ng template DNA (Depending on the primer, after several initial experiments aimed to optimise conditions), $1 \mathrm{X}$ buffer with $\mathrm{MgCl} 2,1 \mathrm{mM}$ $\mathrm{MgCl} 2$, 2.5mM dNTPS, 5pMole Primers, 1 unit Taq DNA polymerase. All the requisite materials were procured from Bangalore Genei, Bangalore, India. The DNA amplification was performed in a thermal cycler (Eppendorf, Germany) programmed to perform initial denaturation at $95^{\circ} \mathrm{C}$ for 7 minutes, followed by 44 cycles and each cycle included denaturation at $94^{\circ} \mathrm{C}$ for 30 seconds, annealing of 45 seconds at $37^{\circ} \mathrm{C}$ for RAPD, extension at $72^{\circ} \mathrm{C}$ for 1 minute and final extension at $72^{\circ} \mathrm{C}$ for 6 minutes. The amplified PCR products were separated on $1.5 \%(\mathrm{w} / \mathrm{v})$ agarose gel containing ethidium bromide $(0.5 \mu \mathrm{g} / \mathrm{ml})$ using $1.0 \%$ tris-acetate EDTA (TAE) buffer and were allowed to run at $100 \mathrm{~V}$ for $45-60$ minutes. Gels were documented by using UV gel documentation system (Vilber Lourmat, Germany).

\section{Molecular data collection and analysis:}

The repeatable and unambiguous RAPD bands were considered for scoring manually and were assigned ' 1 ' or ' 0 ' for presence or absence of bands respectively. The banding pattern derived from primers were used to marker DNA banding characteristics such as total number of bands (TB), number of polymorphic bands (PB) , number of monomorphic bands (MB), percentage of polymorphic bands (PPB), number of monomorphic bands (MB).The discriminatory power of each marker primers was further calculated by four parameters (Varshney et al., 2007) including polymorphic information content (PIC), effective multiplex ratio (EMR), marker index (MI), resolving power (RP).

The genetic data matrix generated from scoring bands was utilized for analysis using MVSP 3.1 software (Kovach, 2007) to generate pairwise genetic similarity matrices by Nei-Li's coefficients (Nei and Li, 1979). GenAlex 6.5 excel add-Ins software (Peakall and Smouse, 2006 and 2012) was used for measurement of genetic diversity indices i.e. Nei's genetic distance (NeiGD), Shannon's Index (I), principal component analysis (PCA) was determined.

\section{STATISTICAL ANALYSIS}

All the measurements were done in triplicates and results are expressed as mean $\pm \mathrm{SD}$. The analysis of variance was performed (ANOVA) by using Origin9 software (OriginLab Corporation, Northampton MA, USA) and Graph Pad Prism 5.01 (Graph Pad Software, San Diego, CA, USA). P values $<0.05$ were considered statistically significant and $\mathrm{P}<0.01$ considered as very significant.

\section{RESULTS AND DISCUSSION}

\section{Quality check and quantitative estimation of isolated DNA}

DNA concentration and absorbance at 260/280 for each sample of Lavatera cachemiriana is mentioned under Table 1. Among all the accessions, the highest DNA yield was found in AHB $(2055 \pm 1.5 \mu \mathrm{g} / \mathrm{ml})$, followed by $\mathrm{DACH}(2025 \pm 0.5 \mu \mathrm{g} / \mathrm{ml})$ and GUL $(2100 \pm 1.85 \mu \mathrm{g} / \mathrm{ml})$. Nevertheless, comparatively lower DNA concentrations were found in PAM and TANG accessions as $1680 \pm 1.0 \mu \mathrm{g} / \mathrm{ml}$ and $545 \pm 1.8$ $\mu \mathrm{g} / \mathrm{ml}$ respectively. The absorbance ratio of DNA at 260/280 was found between 1.74-1.85 which falls closely to the recommended ratio for pure DNA preparations i.e. 1.8-2.0 (Vipranarayana et al., 2012). The agarose gel electrophoresis has revealed distinct, intact and bright DNA bands (Fig.1), which helped to proceed with PCR amplification. The presence of good quality DNA is an essential step for a successful PCR reaction (Ibrahim, 2011) and therefore slightly modified Doyle and Doyle method used by this study could be utilized for routine successful DNA extraction from leaves of Lavatera cachemiriana. 


\section{Molecular marker based polymorphism}

Among 20 primers used, only 15 RAPD primers resulted in scorable and reproducible 137 total bands and with clear gel profiles (Table 2 and Fig 2). The bands were produced in the range of 7-12 with an average of 9.13 bands per primer. Among 137 bands, only 33 were found polymorphic and rest of them were monomorphic. A statistically significant $(p<0.05)$ number of total bands were produced by OPT01 (12), followed by 10 bands from OPA09, OPB05, OPA09, OPT06, further 9 bands each from OPF03, OPM18, OPD18, OPC02, OPB10 and 8 bands were showed by OPG09, OPE20, OPN16, OPD20. The primer-OPA17 could not generate more than 7 scorable bands.

The highest polymorphic bands were generated by OPT06 (9) with percentage of polymorphism as 90\%. Three RAPD primers have showed number of polymorphic bands between 4-6 i.e. OPB10 (4), OPF03/OPG09 (6) with polymorphic percentage between $44.44 \%-75.0 \%$. There were five primers (OPD18, OPE20, OPN16, OPA17 and OPT01) which have showed zero polymorphic bands. The least number of polymorphism was also observed in OPM18, OPB05, OPC02, OPC08, OPD20 primers. The marker parameters of each RAPD primer (PIC, MI, RP) were calculated. Highest RP was observed in OPT06 (3.20), OPF03 (2.80), OPG09/ OPB10 (2.40), OPD20/OPC02 (0.80) and OPC08 (0.40). The primers showed varied MI values between 0-1.90. The highest MI was showed by OPT06 (1.90), followed by OPG09 (1.63), OPF03 (0.89), OPB10 (0.36), OPD20 (0.15), OPC02 (0.07), and OPC08 (0.03). PIC value of each primer was calculated using average value of each loci. PIC value ranged between 0-0.29 with highest value shared by OPT06 (0.23), followed by OPG09 (0.24), OPF03 (0.23), OPB10 (0.16), OPM18/OPC02 (0.07), OPB05 (0.05), 0.08 (OPD20), OPC08 (0.03), the rest of the primers have showed ' 0 ' value of PIC. EMR was found between 0-6.80 with highest value shared by OPG09. Nei's genetic distance (NeiGD) and Shannon's Index (I) were found as 0.119 and 0.075 respectively (Table $3)$.

The Calculated Nei-Li's similarity demonstrated that similarity index ranged between 0.88 - 1 with mean value as 0.964 (Table 4). The genetic relationship of L. cachemiriana sample accessions were analysed using UPGMA algorithm i.e. Unweighted Pair-Group Method of Arithmetic average cluster analysis and the obtained genetic distance has lead construction of dendrogram (Fig 3). Two distinct clusters (1 \& 2) were obtained which formed a major cluster at 0.98 level of similarity. The cluster 1 belongs to TANG and PAMP samples and showed closer genetic relationship with 0.996 level of similarity. Cluster 2 revealed that there does not exist any significant genetic difference between DACH and AHB samples with level of similarity as 1 . The sample accession 'GUL' did not form any cluster and showed similarity level with other clusters at 0.881 .

The knowledge of genetic diversity of threatened and endangered species is an important area to design conservation strategies for their sustainable survival because genetic diversity loss could cause decline of capacity to survive under changing environmental and geographical locations (Lopes et al., 2014). This preliminary study about the evaluation of genetic diversity within populations of Lavatera cachemiriana of Kashmir Himalaya has shred new insights to study its genetic profiling.. The presence of significant but limited levels of genetic differences between the collected accessions of Lavatera cachemiriana. The probable reasons for narrowly distributed plants show lower genetic diversity as compared to species having more geographical distribution (Kumar et al., 2014; Lopes et al., 2014) and results of current study have showed a very close similarity index of 0.97-0.854 (Nei-Li's coefficient) among collected accessions with $17 \%$ of variance among populations. It has been observed by this study that the two samples which were collected from higher altitudes such as DACH and AHB tend to be more related to each other as compared to TANG and PAMP accessions which were collected at lower altitudes. However, one sample accession "GUL" didn't form immediate cluster with any of the accession although it was collected from higher altitude $(10,020$ feet $)$, this indicated that there are other factors responsible for genetic diversity of Lavatera cachemiriana and is not limited to only geographical distance. The existence of genetic diversity showed by the current study seems not solely due to geographical locations because genetic diversity of plant species depends on various factors such as life history, breeding system, growth life forms and method used for evaluation of molecular diversity (Nybom, 2004). The study of in-depth molecular biology of this plant species including its genetic relationship with other species of Lavatera can further ameliorate its conservation and proper 
exploitation including germplasm conservation for sustainable utilization.

\section{ACKNOWLEDGMENTS}

The current work was supported by grant from Sharmila Pharma, Thanjavur, Tamil Nadu and authors are highly obliged.

\section{REFERENCES}

1. Parveen, S. 2013. In vitro studies of some medicinal plants of western Himalayas viz Rheum emodi, Bergenialigulata, Lavatera cashmiriana. Ph.D. Thesis Submitted to University of Kashmir, Srinagar.

2. Dar,G.H., Bhagat, R.C. and Khan, M.A. 2002. Biodiversity of Kashmir Himalaya Valley book house, Srinagar Kashmir, J\&K.

3. Loveless, M.D. and Hamrick, J.L. 1984. Ecological determinants of genetic structure in plant populations. Ann Rev EcolSyst. 15, 65-95.

4. Sundaram, S. and Purwar, S. 2011. Assessment of genetic diversity among fenugreek (Trigonellafoenum-graecum L.), using RAPD molecular markers. J Med Plants Res.5, 15431548 .

5. Domyati, F. M., Younis, R. A., Edris, S., Mansour, A., Sabir, J., Bahieldin, A., 2011. Molecular markers associated with genetic diversity of some medicinal plants in Sinai. $J$. Med. Plants Res. 5, 1918-1929.

6. Chikkaswamy, B.K. and Prasad, M.P. 2012. Evaluation of genetic diversity and relationships in mulberry varieties using RAPD and ISSR molecular markers. Int J Mol Bio. 3, 3.

7. Marin, S. A., Haye, P. A., Marchant, S., Winkler, F. M., 2007. Molecular markers used to analyze species-specific status in abalones with ambiguous morphology. J Shellfish Res. 3, 833-837.

8. Sharma, R. 2003. Medicinal Plants of India, an encyclopedia. DayaPublisihinghouse, Delhi,221222.

9. Kaul, M.K. 1997. Medicinal Plants of Kashmir and Ladhak. Indus PublisihningCo.,New Delhi, 144.

10. SharmaVidyarthi, O.P. 2010. Forest Flora Of Kashmir.Working Plan Circle, Jammu and Kashmir Forest Department, 91-92.
11. Doyle, J.J., Doyle, J.L., 1987. A rapid DNA isolation procedure for small quantities of fresh leaf tissue. Phytochem bull.19, 11-15.

12. Ahmed, N., Nawaz, S., Iqbal, A., Mubin, M., Butt, A., Lightfoot, D. A., Maekawa, M. 2013. Extraction of High-quality Intact DNA from Okra Leaves Despite Their High. Bioscience Methods. 4.

13. Varshney, R. K., Chabane, K., Hendre, P. S., Aggarwal, R. K., Graner, A., 2007. Comparative assessment of EST-SSR, EST-SNP and AFLP markers for evaluation of genetic diversity and conservation of genetic resources using wild, cultivated and elite barleys. Plant Science. 6, 638649.

14. Kovach, W. L., 2007. MVSP 3.1: Multivariate Statistical Package.

15. Nei M., Li, W., 1979. Proc.Natl.Acad.Sci.USA. 74: 5267-5273.

16. Peakall, R., Smouse P.E., 2006. GenAlEx 6: genetic analysis in Excel. Population genetic software for teaching and research. Mol Ecol Notes. 6, 288-295.

17. Vipranarayana, S., Prasad, T. N. V. K. V., Rajinikanth, A., Damodharam, T., 2012. Genomic DNA Isolation and Purification of Two Endemic Medicinal Plants (Pterocarpus Santalinus Linn. F \& Pimpinella Tirupatiensis Bal\& Subr) of Seshachalam Hills, Tirumala. Jbls. 1, 115-120.

18. Ibrahim, R. I. H., 2011. A modified CTAB protocol for DNA extraction from young flower petals of some medicinal plant species. Gene Conserve. 10, 165-182.

19. Lopes, M.S., Mendonca, D., Bettencourt, S., Borba, A.R., Melo, C., Baptista, C. and Machado, A.C. 2014. Genetic diversity of an Azorean endemic and endangered plant species inferred from inter-simple sequence repeat markers. AoB Plants. 1-15.

20. Kumar, A., Mishra, P., Singh, S.C., Sundaresan, V., 2014. Efficiency of ISSR and RAPD markers in genetic divergence analysis and conservation management of Justicia adhatoda L., a medicinal plant. Plant Systematics and Evolution. 6, 14091420 .

21. Nybom, H. 2004. Comparison of different nuclear DNA markers for estimating intraspecific genetic diversity in plants. Mol. Ecol.13, 1143-1155. 
Table 1: Qualitative and Quantitative analysis Lavatera cachemiriana genomic DNA

\begin{tabular}{rccc}
\hline S.No. & Sample Name & $\begin{array}{c}\text { Concentration } \\
(\mu \mathrm{g} / \mathrm{ml})\end{array}$ & A260/280 \\
\hline 1 & AHB & $2055 \pm 1.5$ & 1.85 \\
2 & PAM & $1680 \pm 1.0$ & 1.74 \\
3 & DACH & $2025 \pm 0.5$ & 1.74 \\
4 & GUL & $2100 \pm 1.85$ & 1.8 \\
5 & TANG & $545 \pm 1.8$ & 1.76 \\
\hline
\end{tabular}

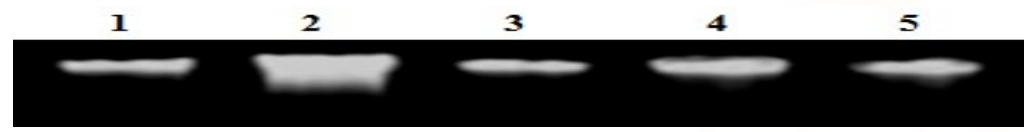

Note: 1-5 indicates sample names AHB, PAM, DACH, GUL and TANG respectively.

Fig 1: Agarose gel (0.8\%) showing quality of DNA extracted from accessions of Lavatera cachemiriana

Table 2: Primers used for RAPD analysis of Lavatera cachemiriana

\begin{tabular}{|c|c|c|c|c|c|c|c|c|c|c|}
\hline S.No. & Primer name & Primer Sequence & IB & MB & PB & PPB (\%) & RP & MI & PIC & EMR \\
\hline 1 & OPGO9 & CTGACGTCAC & 8 & 2 & 6.00 & 75.00 & 2.40 & 1.63 & 0.24 & 6.80 \\
\hline 2 & OPTO6 & CCGTCCCTGA & 10 & 1 & 9.00 & 90.00 & 3.20 & 1.90 & 0.29 & 6.60 \\
\hline 3 & OPF03 & CCTGATCACC & 9 & 3 & 6.00 & 66.67 & 2.80 & 0.89 & 0.23 & 3.86 \\
\hline 4 & OPM18 & TCAGTCCGGG & 9 & 7 & 2.00 & 22.22 & 0.80 & 0.10 & 0.07 & 1.46 \\
\hline 5 & OPA09 & GGGTAACGCC & 10 & 10 & 0.00 & 0.00 & 0.00 & 0.00 & 0.00 & 0.00 \\
\hline 6 & OPB05 & TGCGCCCTTC & 10 & 9 & 1.00 & 10.00 & 0.80 & 0.32 & 0.05 & 6.66 \\
\hline 7 & OPD18 & GAGAGCCAAC & 9 & 9 & 0.00 & 0.00 & 0.00 & 0.00 & 0.00 & 0.00 \\
\hline 8 & OPE20 & AACGGTGACC & 8 & 8 & 0.00 & 0.00 & 0.00 & 0.00 & 0.00 & 0.00 \\
\hline 9 & OPN16 & A.AGCGACCTG & 8 & 8 & 0.00 & 0.00 & 0.00 & 0.00 & 0.00 & 0.00 \\
\hline 10 & OPA17 & GACCGCTTG & 7 & 7 & 0.00 & 0.00 & 0.00 & 0.00 & 0.00 & 0.00 \\
\hline 11 & OPT01 & GGGCCACTCA & 12 & 12 & 0.00 & 0.00 & 0.00 & 0.00 & 0.00 & 0.00 \\
\hline 12 & OPCO2 & GTGAGGCGTC & 9 & 7 & 2.00 & 22.22 & 0.80 & 0.07 & 0.07 & 0.95 \\
\hline 13 & OPCO8 & TGGACCGGTG & 11 & 10 & 1.00 & 9.09 & 0.40 & 0.03 & 0.03 & 0.98 \\
\hline 14 & OPB10 & CTGCTGGGAC & 9 & 5 & 4.00 & 44.44 & 2.40 & 0.36 & 0.16 & 2.28 \\
\hline 15 & OPD20 & ACCCGGTCAC & 8 & 6 & 2.00 & 25.00 & 0.80 & 0.15 & 0.08 & 1.90 \\
\hline
\end{tabular}

Note: TB- total number of bands, MB- monomorphic bands, PB- polymorphic bands, PPB-percentage of polymorphism, RP-resolving power, MI-marker index, PIC-

Polymorphicinformation content, EMR-effective multiplex ratio 
International Journal of Trend in Scientific Research and Development (IJTSRD) ISSN: 2456-6470

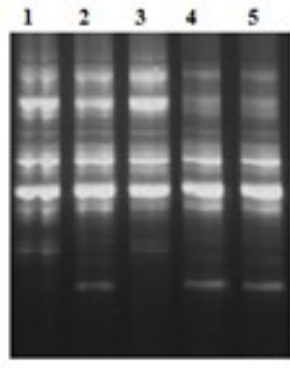

OPF03

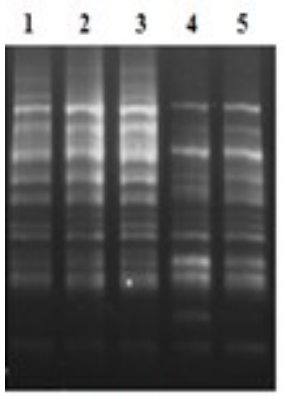

OPT01

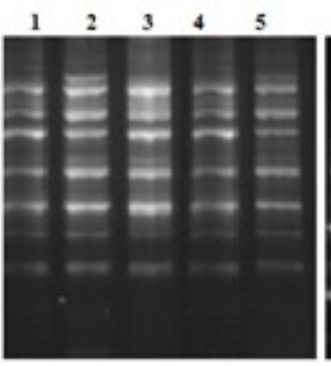

OPMI18

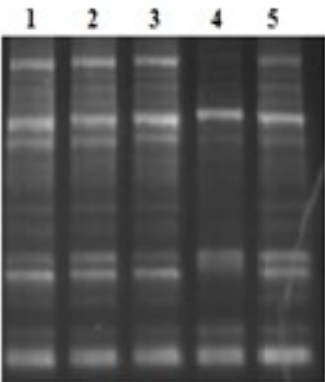

OPC02

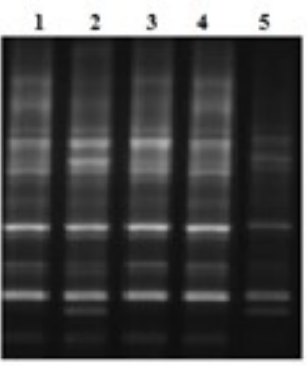

OPB05

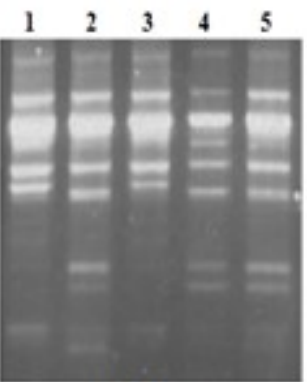

OPB10

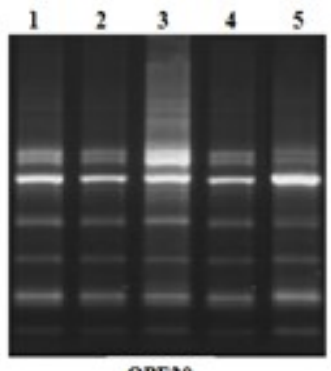

OPE20

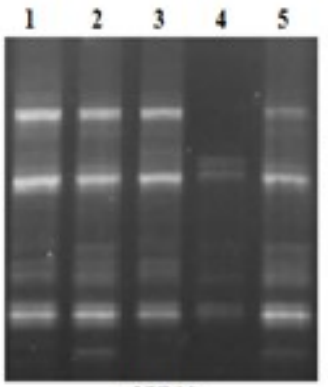

OPD20
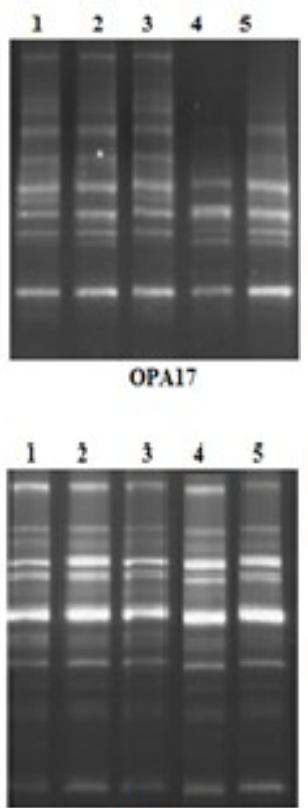

0PT06

Fig 2: Selected RAPD gel profile of Lavatera cachemiriana accessions

Table 3: Genetic Diversity Indices analysed in Lavatera cachemiriana populations using RAPD primers

\begin{tabular}{|c|l|l|l|}
\hline No. of Populations & & I & Nei GD \\
\hline \multirow{2}{*}{2} & Mean & 0.075 & 0.119 \\
\cline { 2 - 4 } & SE & 0.012 & 0.055 \\
\hline
\end{tabular}

$\mathrm{I}=$ Shannon Index; Nei GD= Nei's Genetic Distance.

Table 4: Nei-Li's similarity matrix among accessions of Lavatera cachemiriana based on RAPD data

\begin{tabular}{|l|l|l|l|l|l|}
\hline & AHB & PAMP & DACH & GUL & TANG \\
\hline AHB & 1.00 & & & & \\
\hline PAMP & 0.98 & 1.00 & & & \\
\hline DACH & 1.00 & 0.98 & 1.00 & & \\
\hline GUL & 0.88 & 0.88 & 0.88 & 1.00 & \\
\hline TANG & 0.98 & 1.00 & 0.98 & 0.88 & 1.00 \\
\hline
\end{tabular}

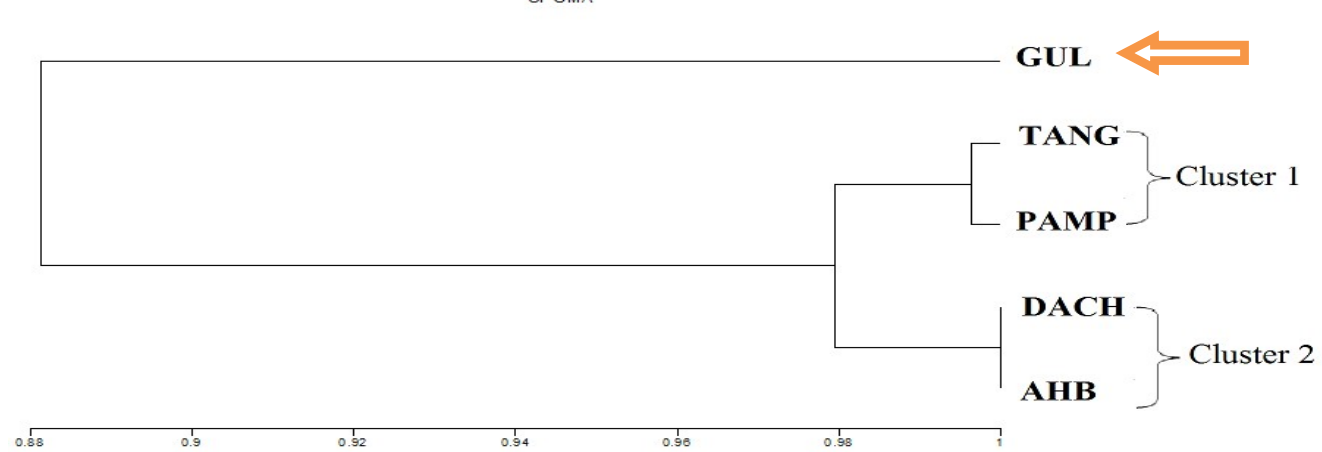

Fig 3: Dendrogram illustrating genetic relationship among different samples of Lavatera cachemiriana using RAPD UPGMA clustering method 